\title{
Subject Review: Iris Recognition Using Neural Network
}

\author{
Haitham Salman Chyad ${ }^{1}$ Raniah Ali Mustafa ${ }^{2}$ \& Rafid Aedan Haleot ${ }^{3}$ \\ ${ }^{1-3}$ Computer Science Department
}

Collage of education, Mustansiriyah University

Baghdad- Iraq

\begin{abstract}
Essentially use iris technique to human identification and recognition. Due enormous growth through the recent years for iris recognition techniques numerous research has emerged in the areas like; image compression, segmentation, quality assessment, Image Acquisition (IA), restoration, feature extraction (FE), normalization, noise reduction iris code matching (NRCM), evaluation, searching large database, applications, performance under changing multibiometrics and condition. Where many techniques were suggested to iris recognition (IR) using neural network because their efficient algorithm and also gives good result. In this research paper, we provide reviews a background for many techniques proposed to recognition iris image in various domains and also procedure a comparison between these techniques.
\end{abstract}

Key Words: Iris recognition system, Neural Network, Feature Extraction, Matching.

\section{INTRODUCTION}

At present there are many of problems for biometric recognition in the world where fake identity for person. This is critical security issue and this is a great challenge to identify the falsify person so the biometric system is a defy to this. There are a lot of biometrics like finger, face, voice, palm etc. but iris is much stable and dependable for identification and extra to fixed and unique thing which doesn't variation with age and also simplicity, speed, precision and applicability these are the several advantageous of iris recognition system [1].

Iris recognition $(\mathrm{RR})$ is one of the most perfect biometric recognition technicality. Nowadays considered Iris recognition is user friendly since the iris can be catches of a specific dimension. crucial stage in the recognition procedure is the partition of the iris pattern in the input eye image. This procedure has to treat with the truth that the iris region of the eye is a comparatively small area, constantly and wet in movement because to automatically eye motions [2].

Artificial neural networks (ANN) or neural networks (NN) for short, are define parallel distributed method, connectionist methods or adaptive methods, due they are consisted by a sequence of interconnectedness treatment elements that work in parallel [3].

Artificial neural networks supply a strong tool to assist doctors to analyses, make and model sensibility composition clinically data across an expansive domain of medical implementations. Most implementations of artificial neural networks (ANN) to medicine are classification troubles; that is, the assignment is on the base of the measured features to specify the patient to one of a small set of classes [4].

In recent years, suggested many of methods for iris recognition due increasing problems for fake identity persons. In this research paper, we work conduct a deep analysis of iris recognition technique that based on neural network (NN). The residual of this research article arranged as follow: In Section 2 the literature survey of some schemes that suggested in the latest decade. In Section 3 comparative analysis of the schemes that discussed in section 2. Finally, the conclusions are shown in Section 4.

\section{LITERATURE SURVEY}

In the recent years, there are many techniques that concerning to iris recognition has been studied. In this section we discuss some of these research. 
In this paper, S.S. Chowhan and G. N. Shinde [5]. performance iris recognition and classification system. The system suggested contains three steps: normalization, feature extraction (FE) and classifier. In this work suggested fuzzy min-max (FMN) neural network classification by Patrick Simpson's for construct utilizes hyperbox fuzzy sets. We utilized CASIA database for 756 images and performed comparison studies of various similarity measurement used to different classifiers. Hence successful probability FMN Classification Algorithm within utilize CASIA database training time with equivalent testing time.

S.Daftry [6]. Used learning vector quantization network (LVQ) and feed forward neural network with back propagation learning (FF) for iris recognition system. The main idea of this paper consist of three phase: the first phase is segmentation and the second phase is 2D discrete wavelet transform (DWT) for extract features through individual wavelet sub bands and the final phase is classification. In this work use two database (Bath database and the UBIRIS database) finds that recognition rates are higher for images from the Bath database depend on quality of the images in which show results $90.00 \%$ recognition rate (RR).

In this article, A. Hilal, B. Daya and P. Beauseroy [7]. Presented an approach novel for iris normalization. In this approach include the stages following: The Daugman's normalization (DN) used parabolic function (PF) and the Gabor filters (GF) used for extract features and the finally stage multilayer perceptron artificial neural network (MPANN) for classification. In this work used CASIA V3 database (DB) iris images. Precision at the equivalent error rate (ER) operating point and receiver operating trait curves offer best results with the parabolic normalization approach and thus suggest its utilize for best iris recognition scheme achievement.

In this paper A. Peter, N. Revathi and M. Mercy [8]. Suggested an efficient algorithm for iris recognition. The system consists of four essential phase. Frist phase utilize Circular Hough Transform (CHT) for perform iris localization. Second phase apply normalize for image into a constant dimension. Three phase is utilizing GLCM textural features and 2D Haar wavelet for extract features (EF). Lastly, artificial Neural Network (ANN) with back propagation (PP) for classification purposes. Iris recognition is extremely efficient because to iris' unique features and the protection of the iris from the aging and environment. Accuracy rate of the suggested algorithm is $97.00 \%$.

In this article, V. S. Raja and S.P. Rajagopalan [9]. Suggested a principle genetic algorithm (GA) for optimization of neural network (NN) for personal identification depend on iris recognition. The method Suggested consists of: Firstly, is performed localization for iris region and then obtain of dataset of iris images. Lastly, perform matching for iris images. in this work results in genetic algorithm better of traditional neural network from where accuracy rate and less error rate.

Iris recognition scheme presented by S. B. Kulkarni, U. P. Kulkarni and S. Tushara M. S. [10] based on UBIRIS database. The scheme proposed consists of stages following: the first stage is utilizing circular Hough transform (CHT) for image segmented and then transformed into fixed sized rectangular block utilizing Daugman's Rubber sheet model (DRSM). The next stage is extracted features for iris utilizing CMYK color model and a feature vector (FV) is generated utilizing 2D Walsh Hadamard transform (2DWHT). The stage final is utilizing Artificial Neural Network (ANN) for perform classification. in scheme is found that the classification rate is $87.1 \%$.

In this research article, K. Hajari, U. Gawande and Y. Golhar [11] Suggested two datasets (CASIA and MMU) for expansion the trustworthy iris recognition framework that can perform in a noisy imaging environmental and to raise the iris recognition rate (RR). In this work suggests two methods: the first a new algorithm for removing noise from the iris image and second algorithm, a texture feature extraction utilizing a composite approach of Gray Level Co-occurrence Matrix (GLCM) and Local Binary Pattern (LBP). Achieved this work highest recognition rate (RR) of (96.5\%) and low error rate and requests minimal performance time.

A novel technique of iris recognition introduced by Y.P. Gautam E. Gautam and S. Tomar [12]. In this work, firstly collected iris images through using CASIA iris database and then after stage image processing extracted features through count the length of iris from left to right and breadth from top to bottom. the stage lastly perform training and testing that use neural network for purpose. The recognition rate (RR) of this technique is $97.5 \%$.

In this paper, A.I. Mozumder and S. A. Begum [13]. Suggests a strategy for recognition of an iris image. in this present work, comprise three steps: the first step is preprocessing (segmentation and normalization based on Quantum-behaved Particle Swarm Optimization (QPSO)) and second step is extracted features from every of the images through using DCT and final step is using Modular Neural Network (MNN) and Fuzzy inference scheme (FIS) based Score Level Fusion (SLF) for purpose recognition of an iris image. The suggested strategy achieved $99.06 \%$ and $98.86 \%$ during identification and verification respectively with the considered dataset. 
In this work, iris recognition system design by W. Zhang and H. Jia [14]. Suggested an approach effective Convolutional Neural Network (CNN). the approach suggested comprise stage preprocess for iris image through extract iris region of the eye. And the stage next, abstract features and identify the iris by divide the iris area into eight rectangular sub-regions are put into the designed model. the stage Final obtain of huge amount of features for purpose iris recognition effective. This work highest recognition rate $(\mathrm{RR})$ of reach about $99.0 \%$.

In this research paper, R. Tobji, W. Di and N. Ayoub [15]. Suggested an algorithm "FMnet" for iris recognition (IR) through utilizing Multi-Scale Convolutional Neural Network (MCNN)and Fully Convolutional Network (FCN). in this work through characteristic of MCNN to learn and work at various resolutions. the approach suggested for recognition the iris conquered the current trouble in the conventional approaches which only utilize handcrafted features extraction (FE), through implementing features extraction (FE) and classification jointly. the algorithm suggested demonstration best classification results as compared to the another state-of-the-art (SOTA) iris recognition method.

\section{COMPARATIVE ANALYSIS OF THE SCHEMES}

In the Table 1 below will demonstrate the comparison between preceding systems.

Table1.1. Comparative analysis of the schemes for some dataset

\begin{tabular}{|c|c|c|c|c|}
\hline Ref. & Dataset & preprocessing and Features & $\begin{array}{l}\text { Recognition } \\
\text { rate }(\mathbf{R R}) \%\end{array}$ & Method used \\
\hline$[5]$ & CASIA & $\begin{array}{l}\text { Singular value decomposition } \\
\text { (SVD) }\end{array}$ & $99.25 \%$ & $\begin{array}{l}\text { fuzzy min-max }(\mathrm{FMN}) \text { neural } \\
\text { network }\end{array}$ \\
\hline$[6]$ & Bath and UBIRIS & $\begin{array}{l}\text { 2D discrete wavelet transform } \\
\text { (DWT) }\end{array}$ & $90.00 \%$ & $\begin{array}{l}\text { vector quantization network (LVQ) } \\
\text { and feed forward neural network }\end{array}$ \\
\hline [7] & CASIA V3 & Gabor Filter(GF) & $97.24 \%$ & $\begin{array}{l}\text { multilayer perceptron artificial neural } \\
\text { network (MPANN) for }\end{array}$ \\
\hline$[8]$ & CASIA & $\begin{array}{c}\text { Circular Hough Transform } \\
\text { (CHT) } \\
\text { GLCM textural features } \\
\text { 2D Haar wavelet }\end{array}$ & $97.00 \%$ & artificial Neural Network (ANN) \\
\hline [9] & CASIA & $\begin{array}{c}\text { circular Hough transform } \\
(\mathrm{CHT}) \\
\end{array}$ & $98.48 \%$ & $\begin{array}{c}\text { genetic algorithm }(\mathrm{GA}) \\
\text { neural network }(\mathrm{NN})\end{array}$ \\
\hline$[10]$ & UBIRIS & $\begin{array}{c}\text { circular Hough transform } \\
(\mathrm{CHT}) \\
\text { 2D Walsh Hadamard transform } \\
(2 \mathrm{DWHT})\end{array}$ & $87.1 \%$ & Artificial Neural Network(ANN) \\
\hline [11] & CASIA and MMU & $\begin{array}{c}\text { Gray Level Co-occurrence } \\
\text { Matrix (GLCM) / Local Binary } \\
\text { Pattern (LBP }\end{array}$ & $96.5 \%$ & Neural Network(ANN) \\
\hline$[12]$ & CASIA & $\begin{array}{l}\text { count the length of iris from } \\
\text { left to right and breadth from } \\
\text { top to bottom }\end{array}$ & $97.5 \%$ & Neural Network(ANN) \\
\hline$[13]$ & IITD & $\begin{array}{c}\text { segmentation and } \\
\text { normalization based on } \\
\text { Quantum-behaved Particle } \\
\text { Swarm Optimization (QPSO) } \\
\text { DCT }\end{array}$ & $\begin{array}{l}99.06 \% \text { and } \\
98.86 \%\end{array}$ & $\begin{array}{l}\text { Modular Neural Network (MNN) and } \\
\text { Fuzzy inference scheme (FIS) }\end{array}$ \\
\hline [14] & CASIA & eight rectangular sub-regions & $99.0 \%$. & $\begin{array}{c}\text { Convolutional Neural Network } \\
(\mathrm{CNN})\end{array}$ \\
\hline [15] & $\begin{array}{l}\text { CASIA-Iris-Thousand, } \\
\text { UBIRIS.v2 and LG2200 }\end{array}$ & handcrafted & $\begin{array}{l}99.41 \% \\
93.17 \% \\
95.63 \% \\
\end{array}$ & $\begin{array}{c}\text { Multi-Scale Convolutional Neural } \\
\text { Network (MCNN) } \\
\text { Convolutional Network (FCN) }\end{array}$ \\
\hline
\end{tabular}




\section{CONCLUSION}

In this research article, we a timeline reviewed various approach for iris recognition depend on neural network within the period (2011-2019). Identity person become very significant security issue specially in security systems. These recognition schemes are studied and analysis wholly to increase the efficiency of the recognition approaches and to warranty the determination identity person. The synopsis of this study, every these the methods are useful for iris recognition. All scheme is unparalleled in its own method, which may be convenient for different applications. Nowadays, the novel iris recognition technology is development so the fast and secure conventional recognition technology will constantly attain a high recognition rate (RR). lately, suggested iris recognition approaches also increase identity person the level of security by providing more than a neural network for iris recognition algorithms. every technologies have several drawbacks and benefits and therefore novel technologies have been sophisticated.

\section{REFERENCES}

[1] G.S. Chandel and N. Bhargava," Identification of People by Iris Recognition", IJCSNS International Journal of Computer Science and Network Security, VOL.14 No.3, March 2014.

[2] Sordo M., "Introduction to Neural Networks in Healthcare", Open Clinical: Knowledge Management For Medical Care, pp. 117, October, 2002.

[3] Gupta A., Shreevastava M., "Medical Diagnosis Using Back Propagation Algorithm", International Journal of Emerging Technology and Advanced Engineering, Vol. 1, issue No. 1, pp 55-58, November, 2011.

[4] S. Daftry, "Artificial Neural Networks based Classification Technique for Iris Recognition", International Journal of Computer Applications (0975 - 8887), Volume 57- No.4, November 2012.

[5] S.S.Chowhan and G. N. Shinde," Iris Recognition Using Fuzzy Min-Max Neural Network", International Journal of Computer and Electrical Engineering, Vol. 3, No. 5, October 2011.

[6] A. Hilal, B. Daya and P. Beauseroy," Improved Iris Recognition using Parabolic Normalization and Multi-layer Perceptron Neural Network", International Joint Conference on Computational Intelligence, DOI: 10.5220/0004155406430646, ISBN: 978989-8565-33-4, 2012.

[7] A. Peter, N. Revathi and M. Mercy,” Neural Network Based Matching Approach for Iris Recognition”, International Journal of Advanced Research in Computer Engineering \& Technology (IJARCET), ISSN: 2278 - 1323, Volume 2, Issue 2, February 2013.

[8] V. S. Raja and S.P. Rajagopalan," IRIS Recognition System using Neural Network and Genetic Algorithm", International Journal of Computer Applications (0975 - 8887), Volume 68- No.20, April 2013.

[9] S. B. Kulkarni, U. P. Kulkarni and S. Tushara M. S.," International Journal of Engineering and Advanced Technology (IJEAT)", ISSN: 2249 - 8958, Volume-3 Issue-5, June 2014.

[10] K. Hajari, U. Gawande and Y. Golhar," Neural Network Approach to Iris Recognition in Noisy Environment”, International Conference on Information Security \& Privacy (ICISP2015), Nagpur, INDIA, Elsevier, 11-12 December 2015.

[11] Y.P. Gautam E. Gautam and S. Tomar," Iris Recognition by Neural Network”, International Conference on Advanced Computing, ISBN-978-93-5288-834-3,2016.

[12] A.I. Mozumder and S. A. Begum,' Iris Recognition Using Modular Neural Network and Fuzzy Inference System Based Score Level Fusion", International Journal of Advanced Research in Computer Science, DOI: http://dx.doi.org/10.26483/ijarcs.v8i7.4260, ISSN No. 0976-5697, Volume 8, No. 7, July - August 2017.

[13] W. Zhang and H. Jia," Research on Convolution Neural Network in Iris Recognition Technology “, 2nd International Conference on Sensor Network and Computer Engineering (ICSNCE 2018), Advances in Computer Science Research, volume 79. 
[14] R. Tobji, W. Di and N. Ayoub," FMnet: Iris Segmentation and Recognition by Using Fully and Multi-Scale CNN for Biometric Security", Appl. Sci. 2019, 9, 2042; doi:10.3390/app9102042.

[15] T. Thomas, A. George and Dr.K P I. Devi,” Effective Iris Recognition System”, Global Colloquium in Recent Advancement and Effectual Researches in Engineering, Science and Technology . Peer-review under responsibility of the organizing committee of RAEREST 2016. 